\title{
Factors Affecting Academic Performance of the Undergraduate Students of Punjab Agricultural University, Ludhiana, India
}

\author{
Anjana Rai* and Kanwaljit Kaur \\ Department of Extension Education and Communication Management, Punjab Agricultural \\ University, Ludhiana, Punjab, India \\ *Corresponding author
}

\begin{abstract}
A B S T R A C T
\end{abstract}
\section{Keywords}

Academic

Performance,

Academic Factors,

University, Students,

Study habits

Article Info

Accepted:

04 August 2018

Available Online:

10 September 2018
The study was conducted to know and compare the factors affecting academic performance of undergraduate students of Punjab Agricultural University, Ludhiana. A sample of 150 students was selected through purposive sampling technique from three Colleges of Punjab Agricultural University, Ludhiana having Government funded programmes namely B.Sc. (Hons.) Home Science, B.Sc. (Hons.) Agriculture, B.Tech. (Agricultural Engineering \& Technology). Data were collected with the help of questionnaire. The findings revealed that 53 percentage of students had medium and 19 per cent had high level of academic performance. The factors like percentage of marks, extra coaching at $10+2$ level and study habits were positively correlated with the academic performance of the students whereas the factors like participation in sports, level of participation in cultural activities and appearing in supplementary and compartment examinations were negatively correlated with the academic performance of the students. Significant association was observed between academic performance of the students who were getting scholarship and involved in student's union activities.

\section{Introduction}

In this era of globalization and technological revolution education is considered as first step for every human activity. It plays vital role in the development of human capital and is linked with well-being and opportunities for better living. It ensures the acquisition of knowledge and skills that enables individuals to increase their productivity which leads towards new sources of earning. This not only enhances the economic growth of a country but also improves the quality of life of each individual. Education is considered to be an important form of national investment for future prosperity and welfare of the nation. Literacy, therefore, is the key unit for developing skills and values in individuals. In our country when rapid technology and economic development is taking place, the academic performance of students is an area of greatest concern which plays an important role in becoming more competent, intelligent and intellectual professionals. Even when these professionals move higher in their life, their academic performance still remains a criterion for deciding and measuring their professional expertise. Academic performance 
at graduation level predicts the future performance of the students for higher education and also for various other vocational courses. Intelligence is not the sole determinant of academic performance. Many other factors contribute or affect the performance of students. The present study was conducted with the following objectives:

To compare academic profile of students of the selected Colleges of PAU.

To study academic factors affecting academic performance of the students.

\section{Materials and Methods}

The study was conducted in three Colleges of PAU, Ludhiana having Government funded undergraduate programmes namely B.Sc. (Hons.) Home Science, B.Sc. (Hons.) Agriculture, B.Tech. (Agricultural Engineering \& Technology). A sample for study was selected through purposive sampling technique. Further 50 students of final year class of each selected programme were selected randomly. Thus total 150 students comprised the sample of study. The data for the study were collected through questionnaire technique.

\section{Operational definitions}

\section{Academic Performance}

It refers to overall credit point average (OCPA) of undergraduate students at end of $7^{\text {th }}$ semester of their respective degree programme. It was divided into three categories i.e., Low (below 7.0), medium (7.08.0) and high (above 8.0 OCPA).

\section{Academic factors}

Academic factors were explored at school as well as University level. At school level i.e.
Year of passing and percentage of marks, stream of education, extra coaching, Board and medium of instruction at $10+2$ level. At University level factors like study habits, availability of College facilities to students, participation of students in sports and cultural activities andtheir level of participation.

\section{Results and Discussion}

Keeping in view the objectives of the study, the academic factors have been discussed in detail and the results have been reported under the following sub- headings.

Academic profile of the students and comparison among students of selected Colleges

Relationship between academic factors and academic performance of the students.

\section{Academic profile of the students}

Academic profile of the students was recorded at School as well as at University level.

Differences of academic profile among the students of selected Colleges were also seen by applying Krushkal wallis $\mathrm{H}$ test.

\section{Percentage of marks at $10+2$ level}

Data in Table 1 revealed that highest percentage $(41.33 \%)$ of students secured above than 70 and below 85 per cent marks at $10+2$ level, whereas 36 per cent students secured above 85 per cent and 22.66 per cent students secured below 70 per cent marks.

Further the higher percentage (44\%) of students of Agriculture College secured above 85 per cent marks while students of College of Home Science $(58 \%)$ and Agricultural Engineering \& Technology (36\%) secured between $70-85$ per cent marks. 


\section{Stream of education at $10+2$ level}

Data given in Table 1 indicated that fifty six per cent of the students had Non- medical as stream of education at $10+2$ level followed by Medical (26.66\%). Least (16\%) percentage of the students was from Arts stream and negligible per cent of students i.e. $0.66 \%$ were from Commerce stream of education at $10+2$ level. Highest percentage of students (90\%) of College of Agricultural Engineering \& Technology had Non-medical as stream of education while, 56 per cent students of Agriculture and 24 per cent of Home Science had Non-medical stream of education at $10+2$ level. Ten per cent students of College of Agricultural Engineering \& Technology had medical stream of education at $10+2$ level because they had Maths as optional subject at $10+2$ level and they were eligible for taking admission in College of Agricultural Engineering and Technology.

\section{Type of school and board of examination at} $10+2$ level

Nearly twenty per cent (19.0\%) had studied in Government school at 10+2 level was 19 per cent, while the majority of the students $(80.7 \%)$ studied in Private schools. Among three Colleges most of the students of College of Home Science $(86 \%)$ had studied from Private schools, on the other hand equal percentage of students (22 per cent) of both i.e. College of Agricultural Engineering \& Technology and College of Agriculture had schooling from Government school at their $10+2$ level.

The percentage of students who had studied from CBSE and State Board at $10+2$ was 71.33 and 26.66 per cent respectively and lowest per cent of students were from ICSE Board. Equal percentage of students of College of Agricultural Engineering and Agriculture i.e. (72\%) had passed their $10+2$ exam from CBSE Board followed by College of Home Science (70\%). Equal percentage of students of College of Agricultural Engineering and College of Home Science (26\%) had passed their exam under state Board and negligible per cent of students were from ICSE Board.

\section{Medium of instruction at $10+2$}

The medium of instruction at $10+2$ level in school was English, Punjabi and Hindi. The large majority (90.7\%) of students had English as medium of instruction, while for Punjabi and Hindi it was eight per cent and one per cent respectively. College wise large majority of Agricultural Engineering \& Technology College (94\%), Home Science College (86\%) and Agriculture College (92\%) had English as medium of instruction at $10+2$ level.

\section{Extra coaching at $10+2$ level}

Data in Table 1 indicated that forty six per cent of students had extra coaching at $10+2$ level. Further percentage of Home Science College students $(22 \%)$ was least who had received any extra coaching at $10+2$ level whereas for Agricultural Engineering \& Technology and Agriculture students it was 60 and 58 per cent respectively.

Significant variation was also seen among students of all Colleges for receiving extra coaching at school level.

\section{Reasons for taking admission in PAU}

Data in Table 1 indicated that sixty eight per cent of students had taken admission in PAU by their own interest and 12.0 per cent students according to their parents' decision whereas 19 per cent students took admission in PAU, Ludhiana because there was no other option. Further College wise reasons expressed by students revealed that students of 
College of Home Science (78\%), Agricultural Engineering (54\%) and Agriculture (74\%) took admission in PAU by their own interest. Equal percentage of Agricultural Engineering \& Technology and Agriculture College students i.e. (10\%) took admission according to their parent's decision whereas highest percentage of Agricultural Engineering students $(36 \%)$ responded that there was no other option.

Krushkal wallis value showed significant variations among students of different Colleges for reasons of taking admission in PAU.

\section{Academic performance}

It is evident from Table 1 that fifty three per cent of the students had medium and 19 per cent of the students had high level of academic performance.

Further College wise data regarding the level of academic performance revealed that highest per cent of students $(66 \%)$ of College of Agriculture had medium academic performance while only 22 per cent students of College of Home Science had high academic performance.

\section{Study habits}

It refers to the habitual practices and study pattern of the students to help them in study, learning and preparing for the test. A check list of ten statements related to study habits of students was prepared. This included main items like whether students studied according to their pre prepared time table, timing of their study i.e. early morning or late night, habits of reading daily, whether students take help from Advisor, seniors, classmates, in difficulties of their study. Each statement was measured on three point continuum i.e. always, sometimes and never.
The total scores of each respondent were calculated by adding the score of the respective statement. Total scores of study habits were divided into three categories i.e. Good (24-27), Average (20-23) and Poor (1619).

It is clear from Table 1 that the forty seven per cent of the students had average study habits (47\%) followed by poor $(32.7 \%)$ and good study habits $(20 \%)$ respectively. Among all three Colleges only 26 per cent of Agricultural Engineering \& technology students had good study habits while other two College i.e. College of Home Science and College of Agriculture percentage in this regard was 18.0 and 16.0 per cent respectively. Sixty four percentage of Agriculture students had average study habits followed by Home Science (46\%) and Agriculture Engineering students (32\%).Poor study habits were also recorded highest in College of Agricultural Engineering (42\%) students followed by Home Science (36\%) and Agriculture students $(20 \%)$.

Significant variation was recorded among students of three different Colleges with regard to their study habits.

\section{College facilities}

Data regarding satisfaction of the students with the facilities which were provided to them by University like library, Wi-Fi, computer, classroom, laboratory, canteen, cafeteria and availability of potable water in College were included in the facilities. Responses were recorded on three point continuum scale. Three, two and one score were assigned to highly satisfactory, satisfactory and unsatisfactory respectively. According to the response of students regarding their satisfaction from College facilities was classified into three categories i.e. highly satisfactory (15-18), satisfactory 
(11-14) and unsatisfactory (7-10). Study results indicated that 35.33 per cent of students were highly satisfied whereas 58.66 per cent students were satisfied and only six per cent students were unsatisfied with College facilities. Further forty four per cent of Agricultural Engineering \& Technology students and 42 per cent Agriculture College students were highly satisfied with the facilities provided to them at their respective College. Only two per cent students of Agriculture and Agriculture Engineering were unsatisfied with the College facilities. Significant variation regarding satisfaction with College facilities was also found among students of three Colleges.

\section{Participation in sports and cultural activities}

Punjab Agricultural University provides great facility and opportunity to the students so that they can show their outstanding performance and their potential in sports and cultural activities.

It refers to number of times student participated in particular activity of sports and cultural events and position obtained by him/ her at College, Inter College, Inter University, State and National level.

Total score $=$ No. of times student participated in particular activity $x$ position obtained $\times$ level of participation

Data in the Table 1 indicated that thirty four per cent of students participated in sports, among these 59 per cent of students had participated at low level whereas only fifteen percentage of student's participation level was high. Among students of three Colleges students of Agricultural Engineering \& Technology had maximum participation (46\%), followed by Home Science (40\%) and least by Agriculture (20\%). The reason for the least participation of Agriculture students in sports may be due to the fact that the students who were selected randomly in the sample for study may not participate in sports.

It was found that one fourth number of students participated in cultural activities (25.33\%) and out of which highest percentage of students had low level of participation $(68.42 \%)$ only 13 per cent students participated at high level.

Significant variation was observed among students of different Colleges in respect of their participation in sports and cultural activities.

\section{Programme option}

Fifty per cent of the students had opted National sports organisation (NSO) programme followed by 48 per cent National Service Scheme (NSS).

The highest percentage of students $(62 \%)$ of Agricultural Engineering \& Technology and Home Science $(56 \%)$ opted National Sports Organisation (NSO), while 62 percentage of Agriculture students opted for NSS. Only two students from College of Agriculture opted National Cadets Cops (NCC).

Non - significant variation was recorded among students of different Colleges for the programme opted during their graduation programme.

\section{Involvement in students' union activities}

It is evident from data in Table 1 that twenty eight per cent of students involved in students' union activities. The involvement was maximum among the students of Agricultural Engineering \& Technology (44\%) while it was minimum among students of Home Science $(18 \%)$. 
Table.1 Distribution of students according to the academic profile at school and university level

\begin{tabular}{|c|c|c|c|c|c|}
\hline Academic Profile & $\begin{array}{c}\text { College of } \\
\text { Agril. Engg. } \\
\mathbf{n}_{1}=50\end{array}$ & $\begin{array}{c}\text { College of } \\
\text { H.Sc. } \\
\mathrm{n}_{2}=50\end{array}$ & $\begin{array}{c}\text { College of } \\
\text { Agri. } \\
\mathbf{n}_{3=50}\end{array}$ & $\begin{array}{l}\text { Total } \\
n=150\end{array}$ & $\begin{array}{c}\text { Krushkal } \\
\text { wallis } \mathbf{H} \\
\text { value }\end{array}$ \\
\hline School level & $\mathbf{f}(\%)$ & $\mathbf{f}(\%)$ & $\mathbf{f}(\%)$ & $\mathbf{f}(\%)$ & \\
\hline $\begin{array}{l}\text { Percentage of marks in } 10+2 \\
\text { Below } 70 \\
70-85 \\
\text { Above } 85\end{array}$ & $\begin{array}{l}12(24.0) \\
18(36.0) \\
20(40.0)\end{array}$ & $\begin{array}{c}9(18.0) \\
29(58.0) \\
12(24.0)\end{array}$ & $\begin{array}{l}13(26.0) \\
15(30.0) \\
22(44.0)\end{array}$ & $\begin{array}{c}34(22.66) \\
62(41.33) \\
54(36.0)\end{array}$ & $4.49^{\mathrm{NS}}$ \\
\hline $\begin{array}{l}\text { Stream of Education at } 10+2 \\
\text { Medical } \\
\text { Non- medical } \\
\text { Arts } \\
\text { Commerce }\end{array}$ & $\begin{array}{c}5(10.0) \\
45(90.0) \\
- \\
-\end{array}$ & $\begin{array}{c}13(26.0) \\
12(24.0) \\
24(48.0) \\
1(2.0)\end{array}$ & $\begin{array}{l}22(44.0) \\
28(56.0) \\
- \\
-\end{array}$ & $\begin{array}{c}40(26.66) \\
85(56.7) \\
24(16.0) \\
1(0.66)\end{array}$ & $2.75^{\mathrm{NS}}$ \\
\hline $\begin{array}{l}\text { Type of school at } 10+2 \\
\text { Government } \\
\text { Private }\end{array}$ & $\begin{array}{l}11(22.0) \\
39(78.0)\end{array}$ & $\begin{array}{c}7(14.0) \\
43(86.0)\end{array}$ & $\begin{array}{l}11(22.0) \\
39(78.0)\end{array}$ & $\begin{array}{l}29(19.33) \\
121(80.66)\end{array}$ & $0.325^{\mathrm{NS}}$ \\
\hline $\begin{array}{l}\text { Name of Board } \\
\text { CBSE } \\
\text { ICSE } \\
\text { State School Education board }\end{array}$ & $\begin{array}{c}36(72.0) \\
1(2.0) \\
13(26.0)\end{array}$ & $\begin{array}{c}35(70.0) \\
2(4.0) \\
13(26.0)\end{array}$ & $\begin{array}{c}36(72.0) \\
- \\
14(28.0)\end{array}$ & $\begin{array}{c}107(71.33) \\
3(2.0) \\
40(26.66)\end{array}$ & $0.119^{\mathrm{NS}}$ \\
\hline $\begin{array}{l}\text { Medium of instruction at } 10+2 \text { level } \\
\text { English } \\
\text { Punjabi } \\
\text { Hindi }\end{array}$ & $\begin{array}{c}47(94.0) \\
3(6.0) \\
-\end{array}$ & $\begin{array}{c}43(86.0) \\
7(14.0) \\
-\end{array}$ & $\begin{array}{c}46(92.0) \\
3(6.0) \\
1(2.0)\end{array}$ & $\begin{array}{c}136(90.66) \\
13(8.66) \\
1(0.66)\end{array}$ & $0.93^{\mathrm{NS}}$ \\
\hline Extra coaching at $10+2$ & $30(60.0)$ & $11(22.0)$ & $29(58.0)$ & $70(46.6)$ & $26.06^{*}$ \\
\hline $\begin{array}{l}\text { Reasons for taking admission in } \\
\text { PAU } \\
\text { Own interest } \\
\text { Parents' decision } \\
\text { No other option }\end{array}$ & $\begin{array}{c}27(54.0) \\
5(10.0) \\
18(36.0)\end{array}$ & $\begin{array}{c}39(78.0) \\
8(16.0) \\
3(6.0)\end{array}$ & $\begin{array}{c}37(74.0) \\
5(10.0) \\
8(16.0)\end{array}$ & $\begin{array}{c}103(68.66) \\
18(12.0) \\
29(19.33)\end{array}$ & $7.93^{*}$ \\
\hline \multicolumn{6}{|l|}{ University level } \\
\hline $\begin{array}{l}\text { Academic performance } \\
\text { Low (below 7.0) } \\
\text { Medium(7.0 -8.0) } \\
\text { High (above 8.0) }\end{array}$ & $\begin{array}{l}19(38.0) \\
23(46.0) \\
8(16.0)\end{array}$ & $\begin{array}{c}15(30.0) \\
24(48.0) \\
11(22.0)\end{array}$ & $\begin{array}{l}7(14.0) \\
33(66.0) \\
10(20.0)\end{array}$ & $\begin{array}{l}41(27.33) \\
80(53.33) \\
29(19.33)\end{array}$ & \\
\hline $\begin{array}{l}\text { Study habits } \\
\text { Good }(24-27) \\
\text { Average }(20-23) \\
\text { Poor }(16-19)\end{array}$ & $\begin{array}{l}13(26.0) \\
16(32.0) \\
21(42.0)\end{array}$ & $\begin{array}{c}9(18.0) \\
23(46.0) \\
18(36.0)\end{array}$ & $\begin{array}{l}8(16.0) \\
32(64.0) \\
10(20.0)\end{array}$ & $\begin{array}{l}30(20.0) \\
71(47.33) \\
49(32.66)\end{array}$ & $23.8^{*}$ \\
\hline $\begin{array}{l}\text { College facilities } \\
\text { Highly satisfactory (15-18) } \\
\text { Satisfactory (11-14) } \\
\text { Unsatisfactory (7-10) }\end{array}$ & $\begin{array}{c}22(44.0) \\
27(54.0) \\
1(2.0)\end{array}$ & $\begin{array}{c}10(20.0) \\
33(66.0) \\
7(14.0)\end{array}$ & $\begin{array}{c}21(42.0) \\
28(56.0) \\
1(2.0)\end{array}$ & $\begin{array}{c}53(35.33) \\
88(58.66) \\
9(6.0)\end{array}$ & $21.2^{*}$ \\
\hline
\end{tabular}


Int.J.Curr.Microbiol.App.Sci (2018) 7(9): 134-146

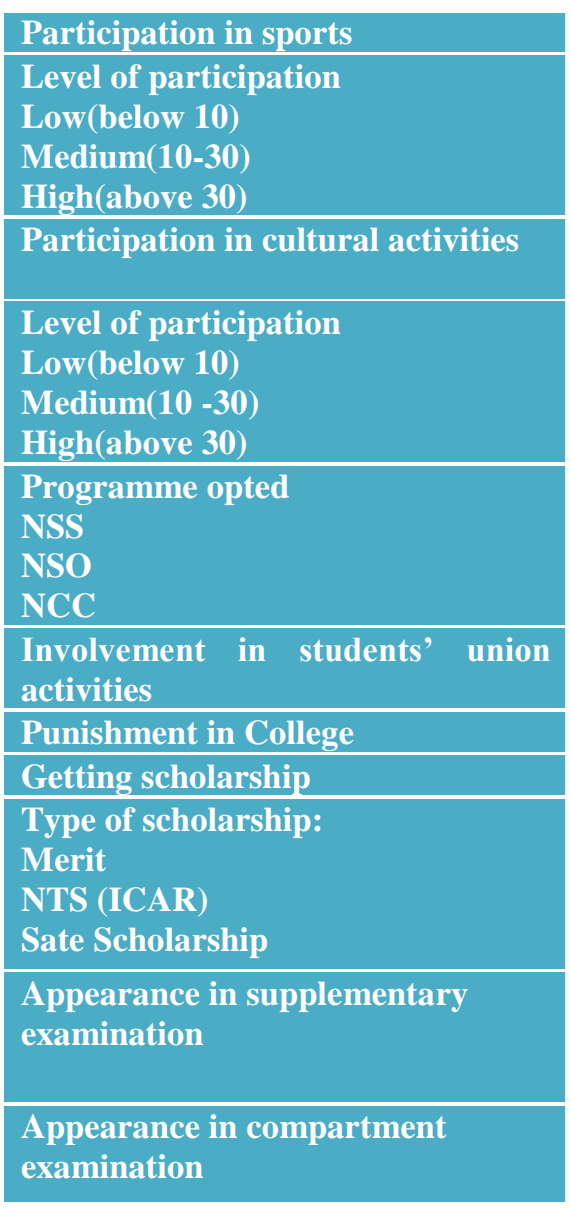

\begin{tabular}{|c|c|c|c|c|}
\hline $23(46.0)$ & $20(40.0)$ & $9(18.0)$ & $52(34.66)$ & $6.18^{*}$ \\
\hline $\begin{array}{c}\mathbf{n}_{\mathbf{1 a}}=\mathbf{2 3} \\
13(56.52) \\
8(34.78) \\
2(8.69)\end{array}$ & $\begin{array}{c}\mathbf{n}_{2 \mathrm{a}}=\mathbf{2 0} \\
14(70.0) \\
3(15.0)) \\
3(15.0)\end{array}$ & $\begin{array}{c}\mathbf{n}_{3 \mathbf{a}}=\mathbf{9} \\
4(44.44) \\
2(22.22) \\
3(33.33)\end{array}$ & $\begin{array}{c}\mathbf{n}_{\mathrm{a}}=\mathbf{5 2} \\
31(59.61) \\
13(25.0) \\
8(15.38)\end{array}$ & $7.89 * *$ \\
\hline $13(26.0)$ & $18(36.0)$ & $7(14.0)$ & $38(25.33)$ & $8.14^{*}$ \\
\hline $\begin{array}{l}\mathbf{n}_{\mathbf{1 a}}=\mathbf{1 3} \\
6(46.15) \\
4(30.76) \\
3(23.07)\end{array}$ & $\begin{array}{c}\mathbf{n}_{2 \mathrm{a}}=\mathbf{1 8} \\
14(77.8) \\
2(11.11) \\
2(11.11)\end{array}$ & $\begin{array}{c}\mathbf{n}_{3 \mathbf{a}}=7 \\
6(85.71) \\
1(11.18) \\
-\end{array}$ & $\begin{array}{c}\mathbf{n}_{\mathrm{a}}=\mathbf{3 8} \\
26(68.42) \\
7(18.42) \\
5(13.15)\end{array}$ & $6.54^{*}$ \\
\hline $\begin{array}{c}19(38.0) \\
31(62.0) \\
\quad-\end{array}$ & $\begin{array}{c}22(44.0) \\
28(56.0) \\
-\end{array}$ & $\begin{array}{c}31(62.0) \\
17(34.0) \\
2(4.0)\end{array}$ & $\begin{array}{c}72(48.0) \\
76(50.6) \\
2(1.33)\end{array}$ & $1.12^{\mathrm{NS}}$ \\
\hline $22(44.0)$ & $9(18.0)$ & $12(24.0)$ & $43(28.66)$ & $5.60^{\mathrm{NS}}$ \\
\hline $13(26.0)$ & $1(2.0)$ & $3(6.0)$ & $17(11.33)$ & $0.43^{\mathrm{NS}}$ \\
\hline $7(14.0)$ & $19(38.0)$ & $15(30.0)$ & 41 (27.33) & $3.15^{\mathrm{NS}}$ \\
\hline $\begin{array}{c}\mathbf{n}_{1 \mathrm{a}}=7 \\
7(100.0) \\
- \\
-\end{array}$ & $\begin{array}{c}\mathbf{n}_{2 \mathrm{a}}=\mathbf{1 9} \\
17(89.4) \\
- \\
2(10.52)\end{array}$ & $\begin{array}{c}\mathbf{n}_{3 \mathrm{a}}=\mathbf{1 5} \\
4(26.7) \\
1(6.7) \\
10(66.6)\end{array}$ & $\begin{array}{c}\mathbf{n}_{\mathbf{a}}=\mathbf{4 1} \\
28(68.29) \\
1(2.43) \\
12(29.26)\end{array}$ & \\
\hline $16(32.0)$ & $14(28.0)$ & $10(20.0)$ & $40(26.66)$ & $1.54^{\mathrm{NS}}$ \\
\hline $8(16.0)$ & $5(10.0)$ & - & $13(8.66)$ & $1.91^{\mathrm{NS}}$ \\
\hline
\end{tabular}

Table.2 Relationship between academic factors at school level and academic performance of the students

$\mathrm{n}=150$

Academic factors

\begin{tabular}{|l|}
\hline At School Level: \\
\hline Percentage of marks in $\mathbf{1 0 + 2}$ \\
\hline Extra coaching at $10+2$ \\
\hline
\end{tabular}

Table.3 Relationship between academic factors at University level and academic performance of the students

Academic performance

\begin{tabular}{|c|c|}
\hline r value \\
\hline $0.308^{* *}$ \\
\hline $0.165^{*}$
\end{tabular}

\begin{tabular}{|c|c|}
\hline & $\mathrm{n}=150$ \\
\hline Academic factors & r value \\
\hline Study habits & $0.205^{*}$ \\
\hline College facilities & $-0.091^{\mathrm{NS}}$ \\
\hline Participation in sports & $-0.146^{*}$ \\
\hline Level of participation in sports & $0.095^{\mathrm{NS}}$ \\
\hline $\begin{array}{l}\text { Participation in } \\
\text { cultural activities }\end{array}$ & $-0.0490^{\mathrm{NS}}$ \\
\hline Level of participation in cultural activities & $-0.184^{* *}$ \\
\hline Supplementary Exam & $-0.514^{*}$ \\
\hline Compartment Exam & $-0.310^{*}$ \\
\hline
\end{tabular}


Table.4 Association between academic factors at school level and academic performance of the students at University level

\begin{tabular}{|c|c|c|c|c|c|}
\hline \multirow{2}{*}{$\begin{array}{l}\text { Academic factors } \\
\text { At school level } \\
\end{array}$} & \multicolumn{4}{|c|}{ Academic performance } & \multirow{2}{*}{$\begin{array}{l}\text { Chi squar } \\
\text { value }\end{array}$} \\
\hline & Low & Medium & High & Total & \\
\hline $\begin{array}{l}\text { Stream of Education } \\
\text { at } 10+2 \\
\text { Non }- \text { Medical } \\
\text { Medical } \\
\text { Arts } \\
\text { Commerce } \\
\end{array}$ & $\begin{array}{c}22(14.6) \\
8(5.33) \\
9(6.0) \\
1(0.66)\end{array}$ & $\begin{array}{c}46(30.66) \\
20(13.3 \\
14(12.0) \\
-\end{array}$ & $\begin{array}{c}17(11.3) \\
12(8.0) \\
- \\
-\end{array}$ & $\begin{array}{c}85(56.66) \\
40(26.6) \\
24(16.0) \\
1(0.66)\end{array}$ & $3.65^{\mathrm{NS}}$ \\
\hline $\begin{array}{l}\text { Type of school at } 10+2 \\
\text { Government } \\
\text { Private }\end{array}$ & $\begin{array}{c}7(4.66) \\
34(22.6)\end{array}$ & $\begin{array}{l}13(8.66) \\
67(44.6)\end{array}$ & $\begin{array}{c}9(6.0) \\
20(13.3)\end{array}$ & $\begin{array}{c}29(19.3) \\
121(80.6)\end{array}$ & $3.15^{\mathrm{NS}}$ \\
\hline $\begin{array}{l}\text { 10+2 pass } \\
\text { Rural } \\
\text { Urban } \\
\end{array}$ & $\begin{array}{c}10(6.66) \\
31(20.66)\end{array}$ & $\begin{array}{c}24(16.0) \\
56(37.33)\end{array}$ & $\begin{array}{l}10(6.66) \\
19(12.6)\end{array}$ & $\begin{array}{l}44(29.33) \\
106(70.6)\end{array}$ & $0.854^{\mathrm{NS}}$ \\
\hline $\begin{array}{l}\text { Medium of instruction at } \mathbf{1 0 + 2} \\
\text { level } \\
\text { English } \\
\text { Punjabi } \\
\text { Hindi }\end{array}$ & $\begin{array}{l}35(23.3) \\
5(3.33) \\
1(0.7)\end{array}$ & $\begin{array}{c}71(47.3) \\
9(6.0) \\
-\end{array}$ & $\begin{array}{c}29(19.3) \\
- \\
-\end{array}$ & $\begin{array}{c}135(90.0) \\
14(9.33) \\
1(0.7)\end{array}$ & $1.42^{\mathrm{NS}}$ \\
\hline $\begin{array}{l}\text { Name of Board } \\
\text { CBSE } \\
\text { ICSE } \\
\text { State School Edu. Board }\end{array}$ & $\begin{array}{c}28(18.7) \\
1(0.7) \\
12(8.0)\end{array}$ & $\begin{array}{c}55(36.7) \\
5(3.33) \\
20(13.33)\end{array}$ & $\begin{array}{l}20(13.3) \\
1(0.7) \\
8(5.33)\end{array}$ & $\begin{array}{c}103(68.6) \\
7(4.66) \\
40(26.7)\end{array}$ & $0.701^{\mathrm{NS}}$ \\
\hline
\end{tabular}

Table.5 Association between academic factors at University level with the academic performance of the students

\begin{tabular}{|c|c|c|c|c|c|}
\hline \multirow[t]{2}{*}{ At University level } & \multicolumn{4}{|c|}{ Academic performance } & Chi square value \\
\hline & Low & Medium & High & Total & \\
\hline $\begin{array}{l}\text { Reasons for taking admission in } \\
\text { PAU } \\
\text { Own interest } \\
\text { Parents' decision } \\
\text { No other option }\end{array}$ & $\begin{array}{c}33(66.0) \\
5(10.0) \\
3(6.0)\end{array}$ & $\begin{array}{l}41(82.0) \\
13(26.0) \\
26(52.0)\end{array}$ & $\begin{array}{c}29(58.0) \\
- \\
-\end{array}$ & $\begin{array}{l}103(68.6) \\
18(12.0) \\
29(19.3)\end{array}$ & $0.004^{\mathrm{NS}}$ \\
\hline $\begin{array}{l}\text { Programme option } \\
\text { NSS } \\
\text { NSO } \\
\text { NCC } \\
\end{array}$ & $\begin{array}{c}15(10.0) \\
25(16.6) \\
1(0.66)\end{array}$ & $\begin{array}{c}38(25.33) \\
41(27.3) \\
1(0.66)\end{array}$ & $\begin{array}{c}19(12.6) \\
10(6.66) \\
-\end{array}$ & $\begin{array}{l}72(48.0) \\
76(50.6) \\
2(1.33)\end{array}$ & $0.191^{\mathrm{NS}}$ \\
\hline $\begin{array}{l}\text { Honour by College } \\
\text { Yes } \\
\text { No }\end{array}$ & $\begin{array}{l}10(6.66) \\
31(20.7)\end{array}$ & $\begin{array}{l}19(12.66) \\
61(40.7)\end{array}$ & $\begin{array}{c}6(4.0) \\
23(15.33)\end{array}$ & $\begin{array}{l}35(23.33) \\
115(76.6)\end{array}$ & $0.928^{\mathrm{NS}}$ \\
\hline $\begin{array}{l}\text { Getting scholarship } \\
\text { Yes } \\
\text { No } \\
\end{array}$ & $\begin{array}{c}4(2.7) \\
37(24.7)\end{array}$ & $\begin{array}{l}21(14.0) \\
59(39.3)\end{array}$ & $\begin{array}{c}16(10.7) \\
13(8.7)\end{array}$ & $\begin{array}{l}41(27.33) \\
111(74.0)\end{array}$ & $20.79 * *$ \\
\hline $\begin{array}{l}\text { Involvement in student's union } \\
\text { activities } \\
\text { Yes } \\
\text { No }\end{array}$ & $\begin{array}{c}18(12.0) \\
23(15.23)\end{array}$ & $\begin{array}{c}20(13.33) \\
60(40.0)\end{array}$ & $\begin{array}{c}5(3.33) \\
24(16.0)\end{array}$ & $\begin{array}{c}43(28.7) \\
107(71.3)\end{array}$ & $9.28 *$ \\
\hline
\end{tabular}

*Significant at $5 \%$ level of significance, $* *$ Significant at $1 \%$ level of significance. 
Score were assigned to positive and negative statements as follows:

\begin{tabular}{|l|c|c|}
\hline Response categories & Scores for Positive statement & Scores for negative statement \\
\hline Always & 3 & 1 \\
\hline Sometimes & 2 & 2 \\
\hline Never & 1 & 3 \\
\hline
\end{tabular}

The scores allotted to different levels of participation and position is given below:

\begin{tabular}{|l|c|}
\hline Level of Participation & Score \\
\hline College level & 1 \\
\hline Inter College level & 2 \\
\hline Inter university level & 3 \\
\hline State level & 4 \\
\hline National level & 5 \\
\hline Position & \\
\hline $\mathbf{1}^{\text {st }}$ & 4 \\
\hline $\mathbf{2}^{\text {nd }}$ & 3 \\
\hline $3^{\text {rd }}$ & 2 \\
\hline No position & 1 \\
\hline
\end{tabular}

Non - significant variation was observed among students of different Colleges for involvement in student's union activities.

\section{Punishment at college level}

Data in Table 1 indicated that very few students $(11 \%)$ were punished during their degree programme. Out of which maximum per cent of students $(26 \%)$ were from College of Agricultural Engineering \& Technology followed by Agriculture students $(6 \%)$ and Home Science (2\%) respectively. Nonsignificant variations were recorded for involvement of students of different Colleges for punishment.

\section{Getting scholarship}

It is clear form data given in Table 1 that only twenty per cent students were receiving scholarship. Among these lowest percentage (14\%) was from Agricultural Engineering \& Technology College. Further highest percentage $(89.4 \%)$ of students of Home Science College who were getting merit scholarship followed by 26 per cent of students of Agriculture College.

Status of supplementary and compartment examination

Data in Table 1 indicated that twenty six per cent students had appeared in supplementary examination during their graduation whereas nine per cent students appeared in compartment examination during their respective degree programme. Appearance of students in supplementary exams among all Colleges was highest among students of College of Agricultural Engineering \& Technology (32\%) and appearance of students in compartment exams was highest found among students of Agriculture (16\%).

\section{Relationship between academic factors and academic performance of the students at University level}

To find out relationship correlation coefficient was worked out for quantitative data and chi square was used for discrete data. 
Data presented in Table 2 showed that the positive correlation was observed between marks obtained in $10+2$ exam and academic performance of the students at their graduation level.

It is clear from the Table 2 that the significant relationship was recorded between extra coaching at $10+2$ level and academic performance of the students at College level.

Silvona and Bray (2006) found that the students who had extra coaching at $10+2$ level performed low in College and contradicted the findings of this study, whereas Lee (2009) found positive and significant relationship between extra coaching at $10+2$ and academic performance at College level and supported the findings of present study.

Data given in the Table 3 revealed the positive relationship between study habits and academic performance of the students. Amin et al., (2014), Jato and Peter (2014), Adesemi and Babutunde (2014) and Cerna (2015) observed similar results in their study that a major cause of underachievement at school was poor study habit and they supported the findings of present study.

It was inferred from data given in Table 3 that there was non- significant relationship observed between two variables i.e. level of satisfaction with availability of College facilities and academic performance of the students. Lawrence (2012) conducted study on school environment and academic achievement of standard IX on 400 students he found no significant relationship between academic performance of standard IX students and school environment and his findings was in agreement with the findings of present study. Devi and Mayuri (2003) and Khan et al., (2012) contradicted the findings of present study and they found significant relationship between academic performance and College facilities provided to the students.

There was significant and negative correlation found between participation in sports and academic performance. But non- significant relationship was found between level of participation in sports and academic performance of the students. Khan et al., (2012) in his study conducted in Government College of District Dera, Ismail khan, Khyber Pakhtunkhwa, revealed that there was link between participation in sports and performance in education and participation in sports improved the Grade Point Average and he contradicted the finding of the present study

Data given in the Table 3 revealed that the coefficient of correlation for participation in cultural activities was non-significant with the academic performance of the students. But negative and significant correlation was observed between level of participation in cultural activities and the academic performance of the students. The reason may be that the students who were participated at high level in cultural activities were devoted more time to cultural activities and less time to their studies. Shamsudin et al., (2014) revealed in their study on 150 students from three public universities in Peninsular Malaysia, indicated that there was no significant association between participation in extra co-curricular activities and student's academic achievements and thus, he supported the findings of present study. On the other hand, Sunita and Annu (2013), Paul and Baskey (2012) indicated that students who participated in co-curricular activities had a marked improvement in the grades. The reasons may be that the attributed to skills they learnt such as better time management to accommodate their hobbies and class activities, better organizational skills and a boost in their self-esteem. Skills learnt in 
clubs such as debate can be applied in the classroom too, as the students learnt how to express themselves better and contradicted the findings of present study.

It was inferred from the data given in Table 3 that low achievers were appeared in supplementary and compartment examination during their whole degree programme. None of the academically high achievers were appeared in supplementary and compartment examination. Negative and significant correlation was also found between appearances of supplementary and compartment examination and level of academic performance of the students.

Association between the academic factors and academic performance of the students at University level.

Data in Table 4 indicated that stream of education, type of school, medium of instruction and type of board at $10+2$ level were non significantly associated with the academic performance of the students at University level. Kolcic and Polasek (2006) concluded that students from urban backgrounds had significantly better academic performance than those from rural backgrounds and contradicted the findings of present study. Considine and Zappala (2006) argued that there was no significant association found between the academic performance of students from rural schools and urban schools and his findings are in agreement with present study.

A critical examination of the data given in Table 5 indicated that there was no significant association found between academic performance of the students and reasons for taking admission in PAU and different kind of programme opted. Johal (2006) also found no association between academic performance and programme opted and supported the findings of present study. A data in Table 5 indicated that there was no association between honour given to the students by College and academic performance of the students. There was significant association found between academic performance of the students who were getting scholarship in the College. It is evident from Table 5 that sixteen per cent of the students who had not any involvement in student's union activities had high level of academic performance. Significant association was also found between academic performance of the students and their involvement in students' union activities at University level.

The academic factor at school level affects the academic performance of the students at University level. Percentage of marks and extra coaching at school level had positive and significant relationship with academic performance at University level. So, there is need to strengthen the instructions at school level for better future performance of the students.

Lowest numbers of Agricultural Engineering and Technology students were getting scholarship as compared to other Colleges and it was further observed that the number of Agriculture College students was higher who were receiving the State scholarship. So, there should be provision of more number of State scholarships to students of other Colleges i.e. College of Home Science and Agricultural Engineering \& Technology

Only twenty per cent students had good study habits. Significant variation in relation to study habits was observed among students of different Colleges.

Further positive and significant relationship was found between study habits and academic performance. So, students should be motivated to develop good study habits. 
Negative and significant relationship was observed between participation in sports and cultural activities during their degree programme and academic performance. So, there is need to guide the students to concentrate in their study along with the participation in extra co-curricular activities.

\section{References}

Adesemi, M., and Babutunde, S. 2014. Personal factors as predictors of student's academic achievement in Colleges of education in southern western, Nigeria. Journal of Academia (8): 97-109.

Amin, Z., Abdulghani, H., and Abdulmajeed, A. 2014. What factors determine academic achievement in high achieving undergraduate medical students? Retrieved from http://www. tandfonline.com.

Cerna, M. 2015. Influence of study habits on academic performance of International College students in Shanghai. Retrieved from http://files.eric.ed.gov/fulltext/ EJ1075118.pdf.

Considine, G., Zappala, G. 2006. Influence of social and economic disadvantage in the academic performance of school students in Australia. Journal of Sociology (38): 129-148.

Devi, S., and Mayuri, K. 2003. The effects of family and School on the Academic Achievement of Residential School Children. Journal of Community Science (2): 139 - 49.

Hawkins, A., 2010. Relationship between undergraduate student activity and academic performance. Retrieved from http://docs.lib.purdue.edu.

Huang, Y., and Chang, S. 2004. Academic and co-curricular involvement: Their relationship and the best combinations for student growth. Journal of College Student Development (4): 391-406.
Jato, S., and Peter, O. 2014. Study habits, use of school libraries and students' academic performance in selected secondary schools in Ondo West Local Government Area of Ondo State. International Journal of Library \& Information Science (6): 57-64.

Johal, K., 2006. The factors affecting academic achievement of undergraduate students of PAU, Ludhiana. M.Sc. Thesis, Punjab Agricultural University, Ludhiana, India.

Khan, M., Jamil, A, Khan A, and Kareem U. 2012. Association between participation in sports and academic achievement of College students. International Journal of Academic Research in Business and Social Science (8): 443-65.

Kolcic, I., and Polasek, O. 2006. Academic Performance and Scientific Involvement of Final Year Medical Students Coming from Urban and Rural Backgrounds. Retrieved from https://www. researchgate.net/publication/7156054.

Lawrence, A., 2012. School environment and academic achievement of standard ix students. Retrieved from https://www.academia.edu.

Lee, K J., 2008. The relationship of eLearner's self-regulatory efficacy and perception of e-Learning environmental quality. Retrieved from https://www. sciencedirect.com/science/article/pii/S0 74756320600152X?via\%3Dihub.

Paul, P., and Baskey, S. 2012. Role of cocurricular activities on academic performance of students: A case study in some secondary schools of Burdwan District in West Bengal, India. International Journal of Innovative Research Development (1): 122-43.

Shamsudin, S., Ismail S, and Mamun, A. 2014. Examining the effect of extracurricular activities on academic achievements among the public 
university students in Malaysia. Journal of Social Sciences (10): 231-37.

Silova, I., and Bray, M. 2006. Education in a hidden marketplace: monitoring of private tutoring. Retrieved from https://www.academia.edu/9621467/mo nitoring of private tutoring.
Sunita, M., and Annu, S. 2013. The relationship between adolescents' participation in extracurricular activities and their academic outcomes. International Journal of Science Research (6): 1340 - 41.

\section{How to cite this article:}

Anjana Rai and Kanwaljit Kaur. 2018. Factors Affecting Academic Performance of the Undergraduate Students of Punjab Agricultural University, Ludhiana, India. Int.J.Curr.Microbiol.App.Sci. 7(09): 134-146. doi: https://doi.org/10.20546/ijcmas.2018.709.018 